\title{
Multi-Path Signal Synchronization Model with Phase Ratio and Dispersion Constraints
}

\author{
Tzu-Jung Huang, Hsun-Jung Cho, Chien-Chia Liäm Huang \\ Department of Transportation and Logistics Management, National ChiaoTung University, Hsinchu, Taiwan 30010 ROC
}

\begin{abstract}
Proper designs of offsets forsignalized intersections in an urban arterial system can help improve the level of service (LoS) significantly. The MAXBAND model has been the most widely used approach for this purpose. In this paper, a MAXBAND model in which the lengths of phases in a cycle are variable is proposed. In other words, the lengths of phases in a cycle, as well as the cycle itself, are no longer fixed.Besides, theincorporation oftraffic dispersion module in the proposed model allows that the travel times from upstream intersection to the downstream one are taken into account.Real-world application on an arterial system in Taiwanfor evaluating the performance of the proposed model is conducted to validate the methodology. Actual traffic flow data are collected through on-site experiments. Results suggest that the improvements are around $44 \%$, on average, in terms of total delay of the entire network.
\end{abstract}

\section{Introduction}

An effective signal control strategyis crucial in improving the LoS for coordinated signalized intersections. By adjusting the offsets of the coordinated intersections in an arterial system, the control delays and the stopping times of vehicles can be well controlled, thereby providing better LoS. The MAXBAND model, proposed by Morgan and Little 1, is one effective approach for this purpose. The concept of the MAXBAND model is to maximizethe total progression bandwidth of a two-way coordinated signalized system by adjusting the offsets at each intersection over atime interval.

The very first MAXBAND model was proposed by Little et al.2 in 1966. The model encompassestime constraintsdescribing timerelationship between the upand down-stream intersections. Chang et al.3proposed the MAXBAND-86 that extendsLittle's model to solve multi-arterial closed network problems. Yet, neithermodel has considered the lead-lag left-turning phases and the queuing clearance times.Gartner et al.4solved the problem by developing a multiband model thatincorporates the left-turn phase sequence optimization and initial queue clearance times.Themajor contribution of multiband model resides in the consideration of the flow volume in the model.

Based on the multiband model, several extensions have been proposed thereafter. Stamatiadis et al.[1]67proposed the Multiband-96 model, extending the multiband model to arterial networks. Lu et al. 8extended the multiband model by incorporating the travel time constraints with traffic dispersion module9. Zhang et al.10improved Gartner's model by relaxing the limitation of symmetrical progression. However, all these models above do not consider the turning flow from the minor legs crossing the arterial. In this case, the efficiency of the arterial systemmay increase, whereas that of the entire network may not necessarily. Most recently, Yang et al.11proposed a multi-path progression model featuring the optimization of the phase sequences and the determination of critical paths in the associated network. This model can handle the intersections with high turning volumes on the minor legs by adding a new path through the arterial.

A major restriction in Yang's model resides in that the length of the common cycle, as well as that of the phases, for the coordinated intersections are fixed.Such a restriction may possibly lead to unsatisfactorylocal solutions. Therefore, based upon this observation, an extension of their model by relaxing such restrictions is proposed so that the lengthsof phases in a cycle, as well as the cycle itself, become variable. Such flexibilities can certainly lead to better solutions, compared to the fixed case. Besides, theincorporation ofRobertson's traffic dispersion module9allows oneto take into accountthe travel times from the upstream intersection to the downstream one, thereby providing more realistic results.Consequently, the resultant model becomes a mixed-integer quadratically constrained programming (MIQCP) problem which is NP-hard 1213 in essence. We then exploit Gurobi solver14to search for an approximate feasible solution. Plus,empirical experiments are conducted for evaluating our methodology, and the results suggest that the performance of our model comes with an improvement being around $44 \%$ on average, in terms of total control delay per minute. 
This is how the rest of the paper is organized. In Section 2, we first review of base model proposed by Yang et al.11, and then introduce the revised model thereafter. In Section 3, we elaborate on how the experiments as well as the numerical simulations are performed. Conclusions will be given in Section 4.

\section{Model Specifications}

In this section, the review of the multi-path progression model proposed by Yang et al.11 is given. Thereafter, tworevised models, one by relaxing the phase ratios in the model, and the other by adding the dispersion module to the model, are introduced.

\subsection{Multi-path progression model - A review}

Variables to be used in the multi-path progression modelare given in Table 1 .

Table 1. Notations

\begin{tabular}{|c|l|}
\hline$P(\bar{P})$ & path sets of outbound(inbound) paths \\
\hline$b_{p}\left(b_{\bar{p}}\right)$ & $\begin{array}{l}\text { total bandwidths of the outbound (inbound) } \\
\text { path } p(\bar{p})\end{array}$ \\
\hline$\varphi_{p}\left(\varphi_{\bar{p}}\right)$ & weight for outbound (inbound) path $p(\bar{p})$. \\
\hline$\beta_{p, l, i}\left(\beta_{\bar{p}, l, i}\right)$ & $\begin{array}{l}\text { binary, equals } 1 \text { if path } p(\bar{p}) \text { is on a green light } \\
\text { in phase } l \text { at intersection } i\end{array}$ \\
\hline$w_{p, i}\left(w_{\bar{p}, i}\right)$ & $\begin{array}{l}\text { the time from the right(left) side of red of path } \\
p(\bar{p}) \text { at intersection } i \text { to the progression line }\end{array}$ \\
\hline$\theta_{i}$ & offset of intersection $i$ \\
\hline$r_{p, i}\left(\bar{r}_{p, i}\right)$ & left- (right-)end red time of the band in a cycle \\
\hline$\tau_{p, i}\left(\tau_{\bar{p}, i}\right)$ & $\begin{array}{l}\text { queue clearance time in the pathpat } \\
\text { intersection } i\end{array}$ \\
\hline$t_{i}\left(\bar{t}_{i}\right)$ & $\begin{array}{l}\text { travel time from intersection } i \text { to } i+1 \text { for } \\
\text { outbound(inbound) link }\end{array}$ \\
\hline$m_{p, i}$ & integer, loop variable \\
\hline$x_{l, m, i}$ & $\begin{array}{l}\text { binary, equals 1 if phase } l \text { precedes phase } m \text { at } \\
\text { intersection } i\end{array}$ \\
\hline$\phi_{l, i}$ & ratio of phase lto a cycle at intersection $i$ \\
\hline
\end{tabular}

The multi-path progression model proposed by Yang et al. 11 is given below

$$
\operatorname{Max} \sum_{p}\left(\varphi_{p} b_{p}\right)+\sum_{\bar{p}}\left(\varphi_{\bar{p}} b_{\bar{p}}\right)
$$

s.t.

$$
\begin{gathered}
w_{p, i}+b_{p} \leq \sum_{l} \beta_{p, l, i} \phi_{l, i} \\
w_{\bar{p}, i}+b_{\bar{p}} \leq \sum_{l} \beta_{\bar{p}, l, i} \phi_{l, i} \\
\theta_{i}+r_{p, i}+w_{p, i}+t_{i}+m_{p, i} \\
=\theta_{i+1}+r_{p, i+1}+w_{p, i+1} \\
+\tau_{p, i+1}+m_{p, i+1} \\
-\theta_{i}+\bar{r}_{\bar{p}, i}+w_{\bar{p}, i}-\tau_{\bar{p}, i}+\bar{t}_{i}+m_{\bar{p}, i} \\
=-\theta_{i+1}+\bar{r}_{\bar{p}, i+1}+w_{\bar{p}, i+1} \\
+m_{\bar{p}, i+1} \\
r_{p, i} \leq \sum_{l} \beta_{p, m, i} x_{l, m, i} \phi_{l, i}+M\left(1-\beta_{p, m, i}\right) \quad \forall m
\end{gathered}
$$

$$
\begin{gathered}
\bar{r}_{p, i} \leq \sum_{l} \beta_{p, m, i} x_{m, l, i} \phi_{l, i}+M\left(1-\beta_{p, m, i}\right) \quad \forall m \\
r_{p, i}+\bar{r}_{p, i}+\sum_{l} \beta_{p, l, i} \phi_{l, i}=1 \\
r_{\bar{p}, i} \leq \sum_{l} \beta_{\bar{p}, m, i} x_{l, m, i} \phi_{l, i}+M\left(1-\beta_{\bar{p}, m, i}\right) \quad \forall m \\
\bar{r}_{\bar{p}, i} \leq \sum_{l} \beta_{\bar{p}, m, i} x_{m, l, i} \phi_{l, i}+M\left(1-\beta_{\bar{p}, m, i}\right) \quad \forall m \\
r_{\bar{p}, i}+\bar{r}_{\bar{p}, i}+\sum_{l} \beta_{\bar{p}, l, i} \phi_{l, i}=1 \\
x_{l, l, i}=0, \quad \forall l \\
x_{l, m, i}+x_{m, l, i}=1, \quad \forall l \neq m ; \forall i \\
x_{l, n, i} \geq x_{l, m, i}+x_{m, n, i}-1, \quad \forall l \neq m \neq n ; \forall i \\
x_{l, n, i}+x_{n, m, i}=1, \forall l \neq m \neq n ; \forall i \\
x_{l, m, i}=1, \quad \forall l \neq m ; \forall i \\
x_{l, m, i}-x_{l^{\prime}, m^{\prime}, i+1}=0, \quad \forall i
\end{gathered}
$$

The decision variables include bandwidth $b_{p}\left(b_{\bar{p}}\right)$, the interference variable $w_{p, i}\left(w_{\bar{p}, i}\right)$, the phase ratio $\phi_{l, i}$, the left(right) side red time $r_{p, i}\left(\bar{r}_{p, i}\right)$, the offset $\theta_{i}$, the loop variable $m_{p, i}$, and the phase relative position variable $x_{l, m, i}$.

The parameters include the weight of bandwidth $\varphi_{p}\left(\varphi_{\bar{p}}\right)$, the available phase parameter $\beta_{p, l, i}\left(\beta_{\bar{p}, l, i}\right)$, the travel time $t_{i}\left(\bar{t}_{i}\right)$, and the queue clearance time $\tau_{p, i}\left(\tau_{\bar{p}, i}\right) . M$ is a penalty parameter.

The objective function (1) is to maximize the sum of bandwidths of outbound $(P)$ and inbound paths $(\bar{P})$. The weights $\varphi_{p}\left(\varphi_{\bar{p}}\right)$ can be adjusted in accordance with the volumes of the associated paths. Here we have $\varphi_{p}=$ $\varphi_{\bar{p}}=1$.

Constraints (2) and (3) are interference constraints. They guarantee that the progression band of path $p(\bar{p})$ will only use the available green time at intersection $i$. Constraints (4) and (5) are loop constraints, representing the relationship of the intersection $i$ and $i+1$ while they are synchronized.

Constraints (6)-(17) are phase sequenceconstraints at each intersection so that the green band competition amongdifferent paths are controlled. Specifically, Constraints (6)-(11) are the boundary conditions for the left/right side red times of progression band. Constraints (12)-(17) ensure that the resulting phase sequence is feasible.

\subsection{Revised model 1: Making $\phi_{l, i}$ a variable}

In the progression model introduced in Section 2, the phase ratio $\phi_{l, i}$ is a constant. Here this restriction is relaxed and $\phi_{l, i}$ is now made a variable. By doing so, constraints associated with $\phi_{l, i}$ become nonlinear in essence. The problem then becomes a nonlinearly constrained mixed integer optimization problem, or more specifically, a mixed integer quadratically constrained programming (MIQCP) problem. Besides,constraints 
(15)-(17) will not be used, similar to the manipulation in Yang et al.11

Along with the relaxation onthe phase ratio, the restriction that the cycle length is also fixed is also relaxed. To do this, we addconstraint (18) to ensure that the cycle length is now varying butbounded.

$$
\frac{1}{e} \leq Z \leq \frac{1}{f}
$$

where $Z$ is the reciprocal of the cycle length, and $e(f)$ is the upper (lower) bound of cycle length.Based on (18), the travel time from intersection $i$ to intersection $i+1$ can then be formulated as

$$
\begin{aligned}
t_{i} & =\frac{d_{i}}{v_{i}} * Z \\
\bar{t}_{\imath} & =\frac{\overline{d_{l}}}{\bar{v}_{i}} * Z
\end{aligned}
$$

where $d_{i}\left(\bar{d}_{i}\right)$ is the distance from outbound (inbound) intersection $i$ to $i+1$; and $v_{i}\left(\bar{v}_{i}\right)$ is the free flow speed of the link.

The sum of the phase lengthsatany intersection must equal the cycle length; in other words, the total ratio of phases at an intersection must equal 1. Thus,

$$
\sum_{l} \phi_{l, i}=1, \forall i
$$

To ensure that the resulting MIQCP is solvable,according to 13 , the model must come withlower boundson the phase ratio variable $\phi_{l, i}$, to begiven in (22). To correctly set the bounds for the ratio variable, we resort to the capacity equation discussed in Roess et al.15, given by

$c_{q}=s_{q} *\left(\frac{g_{q}}{C}\right)$

where $s_{q}$ is the saturation flow rate for lane or lane group $q, g_{q}$ is the effective green time for lane or lane group $q$, and $C$ is signal cycle length. This equation can be rewritten ${ }_{c_{q}} \frac{c_{q}}{s_{q}}=\frac{g_{q}}{c}$. Since the critical volume of lane $V_{q}^{c}$ will never be bigger than the capacity $c_{q}$, we then have $\frac{g_{q}}{C}=\frac{c_{q}}{s_{q}} \geq \frac{V_{q}^{c}}{s_{q}}$. Here, the ratio $\frac{g_{q}}{C}$ can be considered as aproportion of the phase length to the cycle length, which is also the ratio of phase $\left(\phi_{q}\right)$. Consequently, we have $\frac{g_{q}}{C}=\phi_{q}=\frac{c_{q}}{s_{q}} \geq \frac{V_{q}^{c}}{s_{q}}$.

Therefore, the lower bounds for the phase ratio variable $\phi_{l, i}$ are given below

$$
\begin{gathered}
\phi_{l, i} \geq \frac{V_{l, i}^{c}}{s}, \text { if } \sum_{l} \frac{V_{l, i}^{c}}{s}<0.9 ; \\
\phi_{l, i} \geq \frac{V_{l, i}^{c}}{s} *\left(\frac{1}{\sum_{l} \frac{V_{l, i}^{c}}{s}+0.1}\right), \text { otherwise. }
\end{gathered}
$$

The threshold 0.9 is set to evade over-saturation, so that the remaining one-tenth of the entire cycle can be used as further adjustments, if needed.

Therefore, the revisedmodel, termed as RMPPM 1 , is given as follows:

(RMPPM1)Max $\sum_{p}\left(\varphi_{p} b_{p}\right)+\sum_{\bar{p}}\left(\varphi_{\bar{p}} b_{\bar{p}}\right)$

s.t.

Eqs. (2) - (14), (18) - (22)

\subsection{Revised model 2: adding traffic dispersion}

In RMPPM 1 , the parameter $t_{i}$ is mainly defined by the ratio of distance between an intersection to thedownstream $d_{i}$ to the free flow speed of the $\operatorname{link} v_{i}$. In the development of MAXBAND model, there aretwo methods to process $v_{i}$. One is to make it a constant, and the other is to set a boundary for it, see LP2 in Little et al.2. Both methods have their disadvantages. For the first method, the real free flow speed might never be reached even when traffic is moderate to dense; and the second method requires new variables, thereby introducing more complexities to the problem. Based upon this, $\mathrm{Lu}$ et al.8proposed a model that combined the multiband model proposed by Gartner et al.4and the traffic flow dispersion model proposed by Robertson9, so that the calculation of the link travel timecan be more realistic.

In this study, we encompass the suggestion by considering the traffic dispersion module in the proposed RMPPM1, named RMPPM2. The travel time constraints(19)-(20)arethen replacedby(23)-(24)

$$
t_{i}=\sum_{T_{i}=\text { Time }_{\text {fast }}}^{\text {Time }_{\text {slow }}} T_{i} F(1-F)^{T_{i}-\text { Time }_{\text {fast }}} * Z
$$

where $F=\frac{1}{1+0.35 T_{i}}$, Time $_{\text {fast }}=\frac{d_{i}}{V_{\text {fast }}}$, Tim $_{\text {slow }}=\frac{d_{i}}{V_{\text {slow }}}$.

$$
\overline{t_{l}}=\sum_{T_{i}=\sum_{\text {Time }}^{\text {fast }}}^{\overline{T_{\text {tme }} \text { slow }}} T_{i} F(1-F)^{T_{i}-\overline{\text { Trme }_{\text {fast }}}} * Z
$$

where $\quad F=\frac{1}{1+0.35 T_{i}}, \quad \overline{\text { Trme }_{\text {fast }}}=\frac{\overline{d_{l}}}{\overline{V_{\text {fast }}}}, \overline{\text { Trme }}$ slow $=$ $\frac{\overline{d_{l}}}{\overline{V_{\text {slow }}}}$. Time fast $_{\text {fime }}(\overline{\text { fast }})$ is thetravel time of fastest vehicle between intersections $i$ and $i+1$ in an outbound(inbound) path, while Time $_{\text {slow }}\left(\overline{\text { Trme }_{\text {slow }}}\right)$ is thetravel time of slowest vehicle between intersection $i$ and $i+1$ in an outbound(inbound) path.

Consequently, the RMPPM2 is written as the follows: RMPPM2: $\operatorname{Max} \sum_{p}\left(\varphi_{p} b_{p}\right)+\sum_{\bar{p}}\left(\varphi_{\bar{p}} b_{\bar{p}}\right)$

s.t.

Eqs. (2) - (14), (18), (21) - (24)

\section{NUMERICAL EXPERIMENTS}

\subsection{Empirical study}

Areal-world applicationis conducted on the proposed models. The actual flow data of an arterial system,containing a highway ramp and surface signalized intersections, in Chubei City in Taiwan are analysed. Figure 1shows the topology for the underlying arterial system. The traffic data were collected during 17:00 p.m. - 21:00 p.m. in April 13th, 2013. Fivecritical paths are chose for evaluation in accordance with the traffic data. Path 1 contains the traffic flow from the northbound offramp through the major arterial; paths 2 and 3 pass through the major arterial in opposite directions; and paths 4 and 5 are from minor legs of the surface signalized intersections to the major arterial. We mark the three intersections, from right to left, as 1, 2 and 3 , 
respectively.Also, paths 1 and 2 are outbound paths, and paths 3,4 , and 5 are inbound paths.

\subsection{Numerical implementations}

For numerical implementations, both problem solving and traffic simulations for performance evaluations are carried out. For problem solving, a $\mathrm{C}++$ program is coded for connecting theGurobisolver for obtaining a feasible timing plan for the proposed models introduced in Section 2. Obtained signal timing plansthen serve as the input to the traffic software integrated system (TSIS) for random simulations. A total of 500 independent replications are performed and output the associated performance measures.

On-going timing plans as well as those suggested in Yang et al.11are used for comparisons.Designs of phasesat each intersection of the two plansaregiven inTable 2and Table 3.

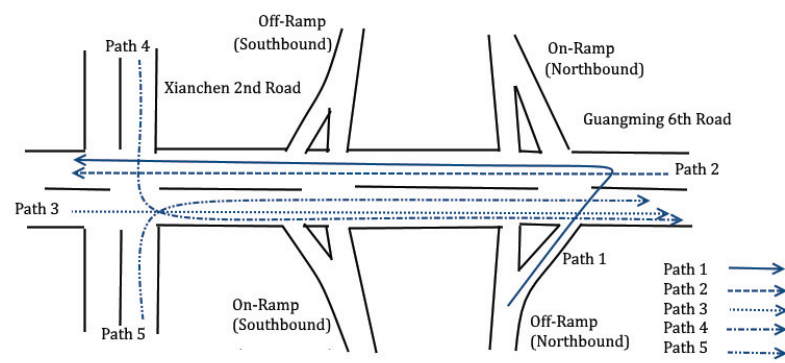

Figure 1.Chubei Network

Table 2.On-going Timing Plan Design

\begin{tabular}{lllll}
\hline \hline On-going plan & Phase 1 & Phase 2 & Phase 3 & Phase 4 \\
\hline Intersection 1 & $\longrightarrow$ & \\
Intersection 2 & $⿱$
\end{tabular}

Table 3.Yang's 11 Timing Plan Design

\begin{tabular}{|c|c|c|c|c|}
\hline Yang's plan & Phase 1 & Phase 2 & Phase 3 & Phase 4 \\
\hline Intersection 1 & & & & \\
\hline Intersection 2 & $\underset{\vec{i}}{\stackrel{亠}{\ddagger}}$ & $\overleftarrow{7}$ & & \\
\hline Intersection 3 & 7 & $\underset{t}{ \pm}$ & & 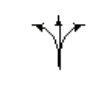 \\
\hline
\end{tabular}

Numerical results are summarized in Table 4andTable 5. The revised results (Plan b, Plan c, Plan y and Plan z) are derived from solving our models. Note that both the phase sequences and cycle lengths differ from the original timing plans (Plan a and Plan $\mathrm{x}$ ).
From Table 4, we see that the cycle length suggested by the proposed model is now longer than the orig inal timing plan. From the timing plan in Plan b, we observe that the lengths of the phase 1'sat intersections 1 and 2 have increased. In particular, this allows more traffic on the major arterial in outbound directions (path 1 and path 2). On the other hand, from the result of Plan c, the cycle length decreases in Plan a,while it increases in Plan b. Also, comparing Plan b and Plan c, the ratio of the phase 1 decreases while the ratio of phase 2 increases.Thissuggests that sacrificing the benefit of outbound pathscan improve that of the inbound paths.

From Table 5, as forthe plan suggested by Yang et al., we can see that comparing the original plan (Plan $\mathrm{x}$ ), the cycle length decreases both in the results of RMPPM 1 (Plan y) and RMPPM 2 (Plan z). For intersection 3 with high right-turn traffic, the model result suggests a longer length of that phase so that the right-turn traffic can dissipate more therein.This can be observed from the results that the ratio of phase 4 at intersection 3 increases. The cycle length of Plan $\mathrm{z}$ is longer than Plan $\mathrm{y}$, but all the phase ratios are closely the same.

\subsection{Performance evaluations}

To evaluatethe system performance under different signal timing plans, we adopt the total control delay per minute in the network as the performance measure.Figure 2 andFigure 3 show the results for the timing plan containing in Table 4 and Table 5, respectively.

Colored lines in Figure 2, from the top to the bottom,correspond tothe total control delay per minute of plans a, b, and c, respectively. It is apparent from Figure 2 that Plan c outperforms the rest plans with respect to the on-going timing plan. Compared to the original signal (Plan a), the improvement with Plan c is around $44.39 \%$ in terms of total control delay per minute of the network; and around $15.59 \%$ compared to plan b.It can be noticed that Plan c, as a matter of fact, comes with the shortest cycle length. We infer that while the cycle length becomes longer, the phase lengthsbecome longer. In this case, at the intersection 1 , the left-turning flow from the westbound will form a queue if the red time is too long, preventing the eastbound left-turning traffic flow at intersection 2 from dissipating.

Lines on Figure 3 are the total control delay per minute of plans $x, y$, and $z$, respectively. Results show that Plan y is the best amongst the rest. Theimprovement is around $59.57 \%$ of delay compared to Plan $\mathrm{x}$; and $13.12 \%$ of delay compared to Plan $z$.

We further compare the performance of each path under the Plan a to Plan c and plan y. This is because Plans $\mathrm{c}$ and $\mathrm{y}$ are respectively the best alternatives derived from solving the RMPPM land RMPPM 2. When we compare Plan c to Plan a, the improvements of the paths are around $20-52 \%$ on average, resulting basically from the improvements on the arterial paths (Path 2 and 3 ). In terms of delay, the improvements are around $37 \%$ $49 \%$. Yet, when compared to Plan y, even though the performance of Path 2 and 3 has improved around 17\%- 
$65 \%$, the performance of the minor paths is less satisfactory, being around $-7 \%-33 \%$.

Table 4. Model Results For On-going Timing Plan

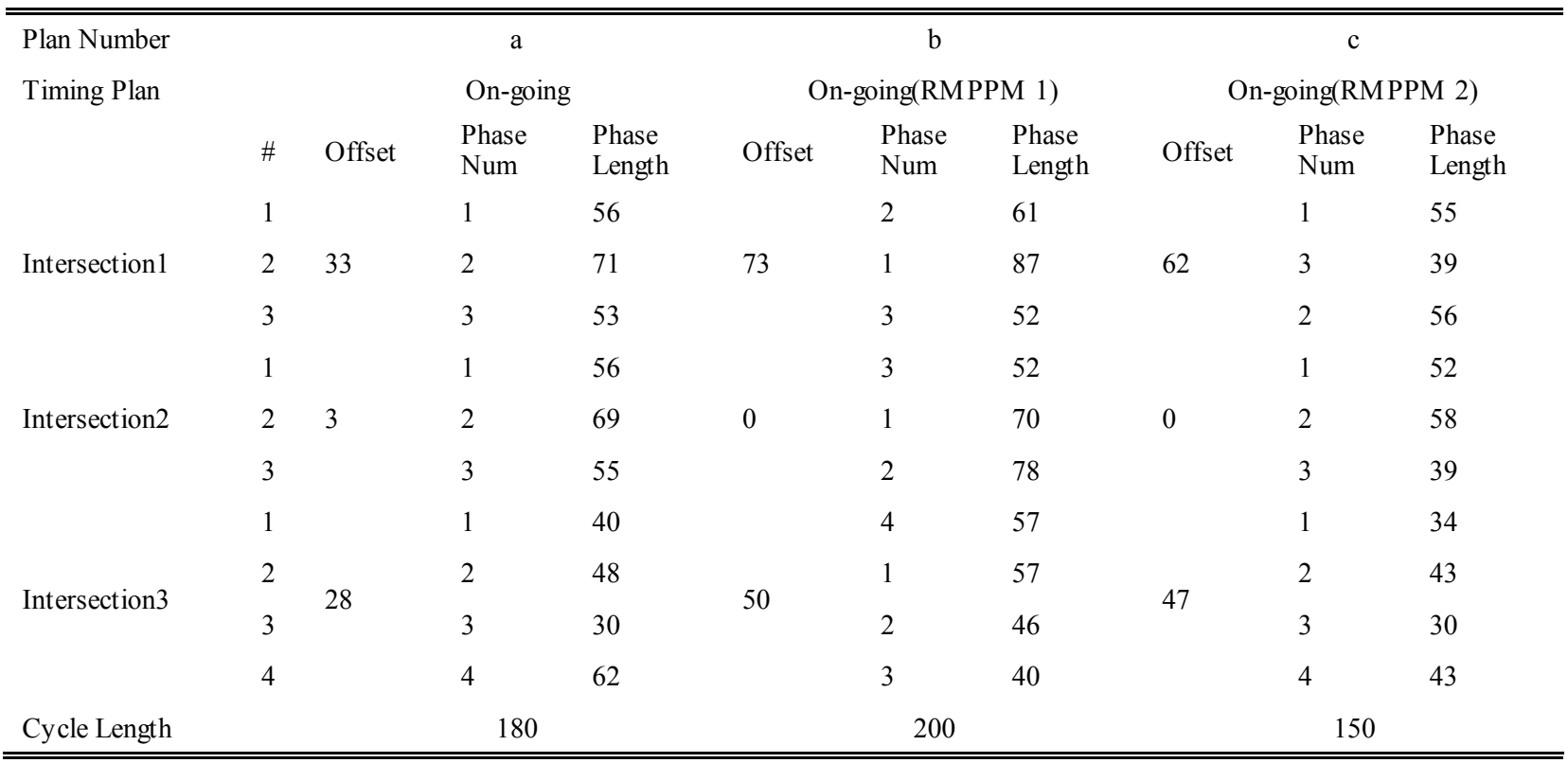

Table 5. Model Results Timing Plan Suggested in Yang et al.11

\begin{tabular}{|c|c|c|c|c|c|c|c|c|c|c|}
\hline \multirow{3}{*}{$\begin{array}{l}\text { Plan Number } \\
\text { Timing Plan }\end{array}$} & & \multicolumn{3}{|c|}{$\mathrm{x}$} & \multicolumn{3}{|c|}{$\mathrm{y}$} & \multicolumn{3}{|c|}{$\mathrm{z}$} \\
\hline & \multirow[b]{2}{*}{$\#$} & \multicolumn{3}{|c|}{ Yang } & \multicolumn{3}{|c|}{ Yang(RMPPM 1) } & \multicolumn{3}{|c|}{ Yang(RMPPM 2) } \\
\hline & & Offset & $\begin{array}{l}\text { Phase } \\
\text { Num }\end{array}$ & $\begin{array}{l}\text { Phase } \\
\text { Length }\end{array}$ & Offset & $\begin{array}{l}\text { Phase } \\
\text { Num }\end{array}$ & $\begin{array}{l}\text { Phase } \\
\text { Length }\end{array}$ & Offset & $\begin{array}{l}\text { Phase } \\
\text { Num }\end{array}$ & $\begin{array}{l}\text { Phase } \\
\text { Length }\end{array}$ \\
\hline \multirow{4}{*}{ Intersection 1} & 1 & & 1 & 89 & & 3 & 66 & & 1 & 51 \\
\hline & 2 & \multirow[t]{3}{*}{0} & 2 & 44 & \multirow[t]{3}{*}{50} & 2 & 39 & \multirow[t]{3}{*}{0} & 3 & 74 \\
\hline & 3 & & 3 & 47 & & 1 & 46 & & 2 & 44 \\
\hline & 1 & & 1 & 79 & & 3 & 39 & & 2 & 66 \\
\hline \multirow[t]{2}{*}{ Intersection2 } & 2 & 3 & 2 & 41 & \multirow[t]{2}{*}{0} & 2 & 58 & \multirow[t]{2}{*}{41} & 1 & 59 \\
\hline & 3 & \multirow{5}{*}{17} & 3 & 60 & & 1 & 52 & & 3 & 44 \\
\hline \multirow{4}{*}{ Intersection3 } & 1 & & 1 & 37 & \multirow{4}{*}{54} & 2 & 43 & \multirow{4}{*}{28} & 4 & 34 \\
\hline & 2 & & 2 & 47 & & 1 & 34 & & 2 & 48 \\
\hline & 3 & & 3 & 68 & & 3 & 43 & & 1 & 39 \\
\hline & 4 & & 4 & 28 & & 4 & 30 & & 3 & 48 \\
\hline Cycle Length & & \multicolumn{3}{|c|}{180} & \multicolumn{3}{|c|}{150} & \multicolumn{3}{|c|}{169} \\
\hline
\end{tabular}




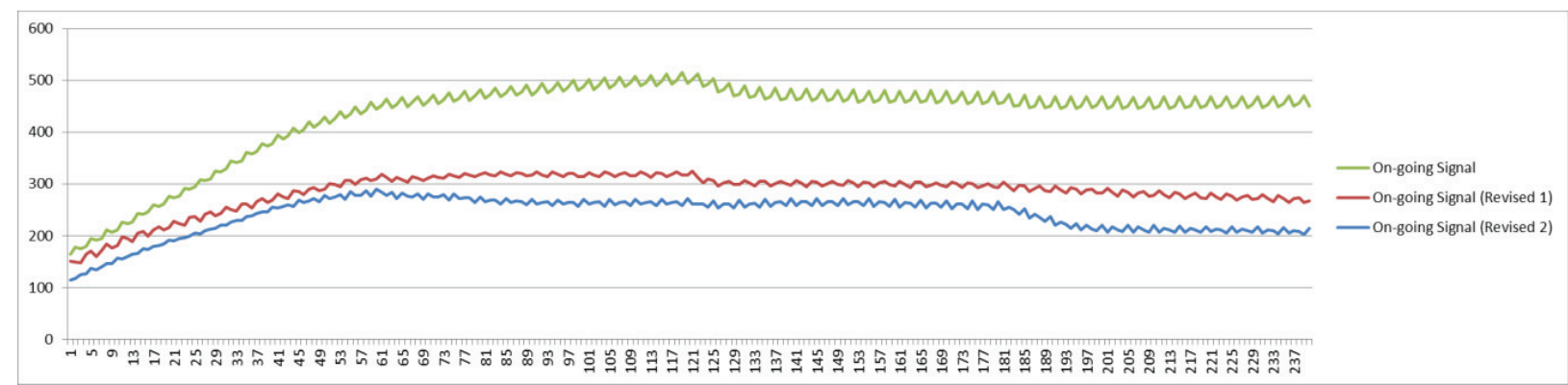

Figure 2. Delay of Control of Chubei Network with On-going Signal Timing Plan

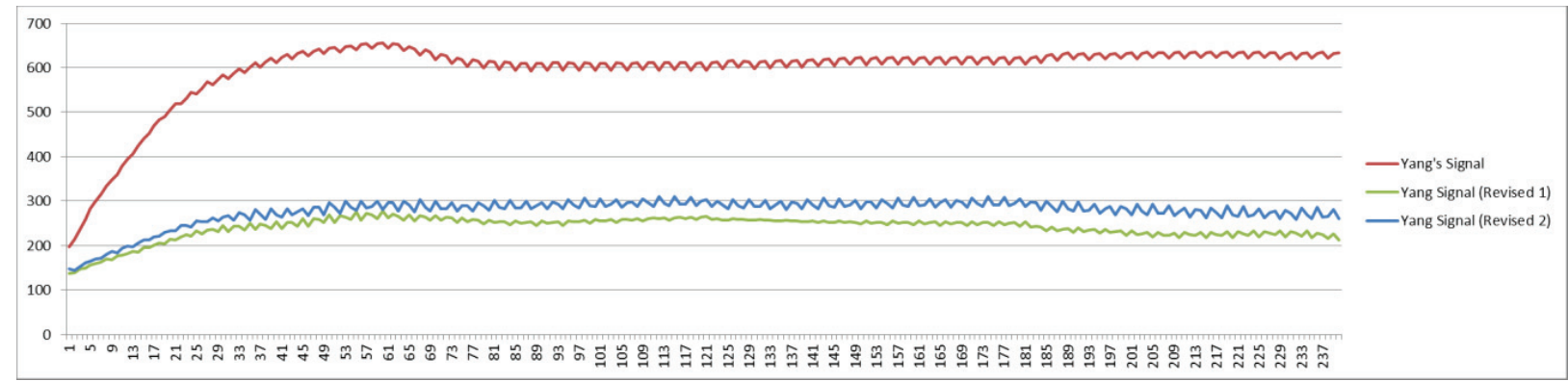

Figure 3.Delay of Control of Chubei Network with Timing Plan of Yang et al.11

Lastly, we compare Plan c to Plan y. From the perspective of path performance, Plan $\mathrm{c}$ is around 10\% better than Plan y with respect to Paths 1 and 5. Yet, on the other hand, Plan y yields better results on Path 3. As to Paths 2 and 4, Plan y is better. Despite this fact, Plan c is the best alternative in terms of the aggregate network delay, possibly due to the lack of efficiency in the turning flow at intersection 3 under Plan y.

\section{Conclusions}

In this paper, extensions of the signal synchronization model proposed in Yang et al.11are given by letting the ratios of phases become variable, and by incorporating the traffic flow dispersion module for adjusting the travel times. Numerical evidences suggest a total improvement of around $44.39 \%$ and $59.57 \%$ with on-going timing plan and those suggested by Yang et al.11, respectively, in terms of the total control delay per minute of the entire network. We observe that in this experiment, the results of RMPPM 2 are not always better than that of RMPPM 1. This may be due to interference of the paths that we have not considered in the experiment - such as the path flow from the northbound of intersection 2 and the leftturning flow at intersection 2 - or the red time is too longer to remove the queue in each cycle and keep the progression.

Comparisons on the performance of each path under different plans are carried out as well. Results show that compared to the on-going timing plan (Plan a), with Plan c, the total control delay of each path can be improved around $20 \%$ to $52 \%$; with Plan y, the total control delay of paths mainly in the major, such as path 2 and path 3 , will be improved around $17 \%$ to $65 \%$. But when it comes to paths through the branches, the results of our model have a smaller contribution for the total control delay of them, due to the difference of the signal design.
As of this moment, discussions of the signal synchronization problem still are limited by assuming that the design of the phases comprising a cycle is predefined. A potential future research direction resides in the optimal design of phases for signalized intersections. A proper design of phases can certain help improve the LoS of both the underlying signalized intersections and the entire network associated.

\section{Acknowledgment}

This study is supported by Ministry of Science and Technology (MOST), Taiwan, with Grant No. MOST105C581; and Chunghwa Telecom Laboratories, Taiwan, with Grant No. 105R951, with Department of Transportation and Logistics Management, National ChiaoTung University.

\section{References}

1. Morgan, J. T. and Little, J. D. C., "Synchronizing Traffic Signals for Maximal Bandwidth," in Operations Research, 12 (6), pp. 896-912 (1964).

2. Little, J. D. C., "The Synchronization of Traffic Signal by Mixed-Integer Linear Programming," in Operations Research, 14 (4), pp. 568-594 (1966).

3. Chang, Cohen, Liu, Chaudhary, and Messer, "MAXBAND-86: program for optimizing left-turn phase sequence in multi-arterial closednetworks," in Transportation Research Record, 1181, pp. 61-67 (1998).

4. Gartner, N. H., Assman, S. F., Lasaga, F., and Hou, D. L., "A Multi-Band Approach to Arterial Traffic Signal Optimization," in Transportation Research Part B, 25 (1), pp. 55-74 (1991). 
5. Stamatiadis, C., Gartner, N.H., "MULTIBAND-96: a program for variable-bandwidth progression optimization of multiarterial traffic networks,"in TransportationResearch Record, 1554, pp. 9-17 (1996).

6. Gartner, N.H. and C. Stamatiadis, "Arterial-Based Control of Traffic Flow in Urban Grid Networks," in Mathematical and Computer Modelling, 35, pp. 657-671 (2002).

7. Gartner, N.H. and C. Stamatiadis, "Progression Optimization Featuring Arterial- and Route-Based Priority Signal Networks," in Journal of Intelligent Transportation Systems, 8, pp. 77-86 (2004).

8. Lu, S., Liu, X., and Dai, S., "Revised MAXBAND Model for Bandwidth Optimization of Traffic Flow Dispersion,"in 2008 ISECS International Colloquium on Computing, Communication, Control, and Management, pp. 85-89 (2008).

9. Robertson, "TRANSYT: a traffic network study tool, Road Research Laboratory Report", LR 253. Road Research Laboratory, Crowthorne (1969).
10. C. Zhang, Y.Xie, N. H. Gartner, C.Stamatiadis, and T.Arsava, "AM-Band: An Asymmetrical MultiBand model for arterialtraffic signal coordination ," in Transportation Research Part C, 58, pp. 515-531 (2015).

11. Yang, X., Cheng, Y., and Chang, G. L. "A multipath progression model for synchronization of arterial traffic signals," in Transportation Research Part C: Emerging Technologies, 53, pp. 93-111 (2015).

12. P. Pardalos, "Global optimization algorithms for linearly constrained indefinite quadratic problems," in Computers and Mathematics with Applications, 21, pp. 87-97 (1991).

13. R. Jeroslow, "There cannot be any algorithm for integer programming withquadratic constraints," in Operations Research, 21, pp. 221-224 (1973).

14. Optimization, G. (2012). "Gurobi optimizer reference manual", $2,1-3$. Retrieved Oct. $31^{\text {th }}, 2016$, from http://www.gurobi.com.

15. Roess, R. P., Prassas, E., andMcShane, W. R.,"Traffic Engineering", Pearson Education Inc.(2004) 